\title{
Spin torques in ferromagnetic/normal-metal structures
}

\author{
K. Xia, ${ }^{1}$ P. J. Kelly, ${ }^{1}$ G. E. W. Bauer, ${ }^{2}$ A. Brataas,${ }^{3}$ and I. Turek ${ }^{4}$ \\ ${ }^{1}$ Faculty of Applied Physics and $\mathrm{MESA}^{+}$Research Institute, University of Twente, P.O. Box 217, 7500 AE Enschede, The Netherlands \\ ${ }^{2}$ Department of Applied Physics and DIMES, Delft University of Technology, Lorentzweg 1, 2628 CJ Delft, The Netherlands \\ ${ }^{3}$ Harvard University, Lyman Laboratory of Physics, Cambridge, Massachusetts 02138 \\ ${ }^{4}$ Institute of Physics of Materials, Academy of Sciences of the Czech Republic, CZ-616 62 Brno, Czech Republic
}

(Received 28 March 2002; published 23 May 2002)

\begin{abstract}
Recent theories of spin-current-induced magnetization reversal are formulated in terms of a spin-mixing conductance $G^{m i x}$. We evaluate $G^{m i x}$ from first principles for a number of (dis)ordered interfaces between magnetic and nonmagnetic materials. We predict that the magnetization direction of a ferromagnetic insulator or of one side of a tunnel junction in a multiterminal device can be switched even though a negligible charge current is passed.
\end{abstract}

DOI: 10.1103/PhysRevB.65.220401

PACS number(s): 72.25.-b, 71.15.Ap, 73.21.Ac

"Giant magnetoresistance" refers to the large change of resistance brought about by applying an external magnetic field to change the angle between the magnetization directions of magnetic films separated by nonmagnetic spacers. ${ }^{1}$ Since a spin injected into a magnetic material experiences a torque, it has been argued that passage of a current through adjacent magnetic layers should lead to the transfer of spinangular momentum from one layer to the other ${ }^{2,3}$ with possible reorientation of the magnetizations for sufficiently large currents. ${ }^{2}$ Interestingly, the sign of the corresponding torque should be reversed on changing the current direction leading to the possibility of making an electronically accessible nonvolatile magnetic memory whose performance on downscaling compares favorably with other alternatives. ${ }^{4}$ Promising proof-of-principle experiments on current-induced magnetization reversal ("spin transfer") have been carried out ${ }^{5}$ but the large current densities required underline the need to optimize the effect.

Theoretical discussion of the giant magnetoresistance and tunneling magnetoresistance (TMR) effects in collinear spin systems is greatly simplified when the spin-flip scattering is so weak that it is possible to consider the spin-up and spindown conduction channels separately. ${ }^{1}$ When studying current-induced magnetization reversal it is necessary to consider what happens when the spin-quantization axis rotates on going from one material to another; the current operator then has to be represented in a $2 \times 2$ spin space even when spin-flip scattering is neglected entirely. Slonczewski ${ }^{2}$ used a model of free electrons incident on a spin-dependent potential barrier to discuss the qualitative aspects of spin transfer. Free-electron models are known to miss an important contribution to spin transport in layered magnetic materials coming from the mismatch of the complex $d$ bands responsible for itinerant ferromagnetism, ${ }^{6,7}$ and it is important to take this into account. A more general framework, suitable for treating complex band structures and for including the effects of disorder, is provided by the scattering theoretical formalism of Waintal et al. ${ }^{8}$ The "circuit theory" of Brataas et al. ${ }^{9}$ is nearly equivalent, but is more transparent and flexible, making the treatment of many-terminal devices straightforward. In this circuit theory, the torque and current are formulated in terms of (real) spin-up and spin-down conductances
$G^{\uparrow}$ and $G^{\downarrow}$, and a new spin-mixing conductance $G^{m i x}$ which is complex. The mixing conductance has only been studied using free-electron models and little is known about its dependence on real material parameters. In this paper we focus on how $G^{m i x}$ depends on typical materials used.

To evaluate the spin-up, spin-down, and spin-mixing conductances for systems of current interest we use methods ${ }^{10-12}$ recently developed to calculate the scattering matrix $^{13}$ within the framework of density-functional theory. We show that the spin-mixing conductance in tunnel junctions can remain large even when the conventional conductance itself is made vanishingly small. This result is unexpected and may offer important advantages for the application of spin-torque effects. A spin current can be injected in the absence of an electron current using the threeterminal device sketched in Fig. 1(a). ${ }^{9}$ FM, FM1, and FM2 are ferromagnetic circuit elements, NM is nonmagnetic. A current from FM1 into FM2 induces a spin accumulation in the NM node. For a given spin accumulation, the torque on FM is determined only by the mixing conductance. We shall therefore not calculate the spin accumulation explicitly but simply assume that it exists in NM and analyze what happens when FM is a magnetic insulator or is the top magnetic element of a magnetic tunnel junction. In the latter case it is possible to independently determine the orientation of FM by measuring the TMR. ${ }^{14}$ The spin torque is that of the metallic junction, but without the energy dissipation caused by the particle current. In practical memory devices it may be advantageous to be able to achieve this separation of particle and spin injection.

We begin with the two basic elements of a magnetic circuit: a nonmagnetic element NM in which there is a spin accumulation in the direction $\mathbf{s}$ (as a result of a source-drain current from FM1 to FM2) and a ferromagnetic element FM whose magnetization is given by the unit vector $\mathbf{m}$; see Fig. 1(a). In general $\mathbf{s}$ and $\mathbf{m}$ are noncollinear and it is convenient to split the $2 \times 2$ matrix current $\hat{I}$ from the nonmagnetic element into the ferromagnetic element into a scalar charge current $I_{0}$ and a vector spin current $\mathbf{I}_{s}: \hat{I}=\left(I_{0}+\boldsymbol{\sigma} \cdot \mathbf{I}_{s}\right) / 2$, where $\boldsymbol{\sigma}$ is a vector of Pauli spin matrices. When a spin current is injected into a ferromagnetic material, the component of $\mathbf{I}_{s}$ perpendicular to the magnetization direction $\mathbf{m}$ (times the 

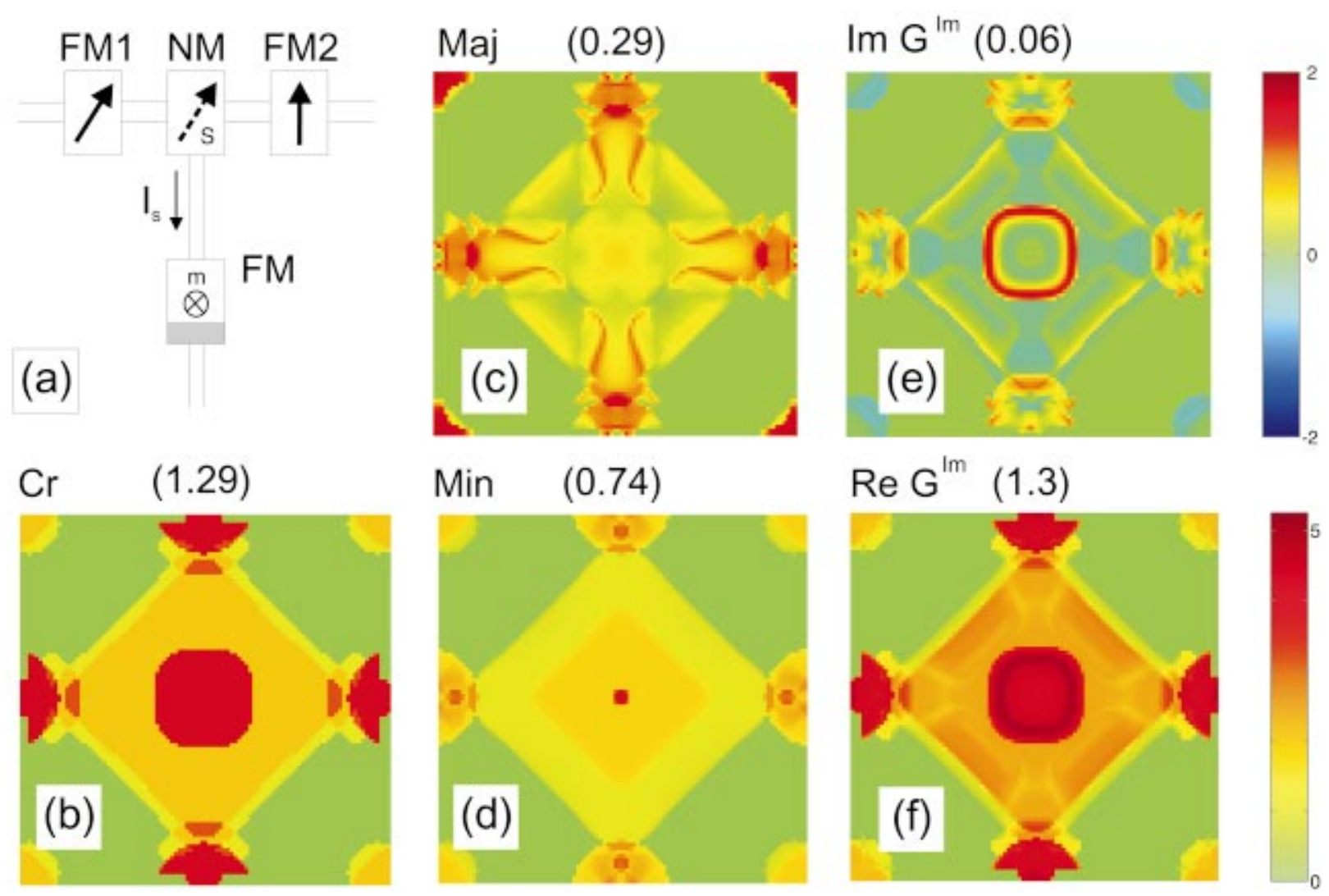

FIG. 1. (Color) (a) Sketch of a three-terminal device where a normal metal (NM) element is connected to three ferromagnetic elements FM1, FM2, and FM. An applied bias causes a current to flow between FM1 and FM2. If FM is a tunnel junction or a magnetic insulator then the particle flow into FM will be vanishingly small. (b) Number of propagating channels in first Brillouin zone for bulk Cr. For a clean Cr/Fe interface (c) and (d) show the majority-spin and minority-spin conductances, (e) and (f) the imaginary and real parts of the mixing conductance. Units of conductance are $e^{2} / h$. The result of integrating over the whole Brillouin zone is given in brackets at the top of each panel. Note that the color scale in the top row differs from that in the bottom row but zero is represented by the same (green) color.

Bohr magneton and gyromagnetic ratio) equals the torque acting on the ferromagnet ${ }^{8}$ and this is determined entirely by the real and imaginary parts of $G^{\text {mix }} \cdot{ }^{15}$ The spin-transfer effect can be most readily understood when the magnetic layers are perfect spin filters, i.e., when spins aligned (anti)parallel to the magnetization of a layer are completely (reflected) transmitted. Since spin-angular momentum is globally conserved, the remaining spin component must be transferred to the scattering layer.

The spin-up, spin-down, and spin-mixing conductances characterizing transport through an interface are defined in terms of the spin-dependent transmission and reflection matrices ${ }^{16} t_{\uparrow(\downarrow)}$ and $r_{\uparrow(\downarrow)}$ (the latter as seen from the normal metal) $\quad$ as $\quad G^{\uparrow}=\left(e^{2} / h\right) \operatorname{tr}\left(t_{\uparrow}^{\dagger} t_{\uparrow}\right), \quad G^{\downarrow}=\left(e^{2} / h\right) \operatorname{tr}\left(t^{\dagger} t_{\downarrow}\right)$, and $G^{m i x}=\left(e^{2} / h\right) \operatorname{tr}\left(I-r_{\uparrow}^{\dagger} r_{\downarrow}\right) . I$ is an $M \times M$ unit matrix where $M$ is the number of conducting channels in the $\mathrm{NM}$ element. ${ }^{8,9}$

The parameter-free calculation of the transmission and reflection matrices ${ }^{12}$ is based on the surface Green's-function method ${ }^{17}$ implemented with a tight-binding linear muffin-tin orbital basis. ${ }^{18}$ Because a minimal basis set is used, we are able to perform calculations for lateral supercells containing as many as $10 \times 10$ atoms and to model disorder very flexibly within such supercells without using any adjustable param- eters. The electronic structure is calculated self-consistently within the local spin density approximation. For disordered layers the potentials are determined using the layer coherentpotential approximation (CPA) approximation. ${ }^{19}$ The calculations are carried out with a $\mathbf{k}_{\|}$mesh density equivalent to more than 3600 mesh points in the two-dimensional Brillouin zone (BZ) of a $1 \times 1$ interface unit cell. The results of calculations for clean and disordered fcc(111) $\mathrm{Cu} / \mathrm{Co}$ and bcc(001) $\mathrm{Cr} / \mathrm{Fe}$ metallic interfaces and for an fcc(111) $\mathrm{Cu} /$ $\mathrm{Co} / \mathrm{Vac} / \mathrm{Co}$ tunneling configuration are given in Table I.

We first discuss our results for the $\mathrm{Cu} / \mathrm{Co}$ and $\mathrm{Cr} / \mathrm{Fe}$ interfaces. Both have been the subject of much study in the context of exchange coupling and giant magnetoresistance and different calculations of the interface transmission matrices yield very similar results for the spin-dependent interface resistances. ${ }^{10-12}$ The atomic volume of each pair of materials is very similar and we neglect the difference here. The disordered interfaces are simply modeled as semi-infinite $\mathrm{Cu} 2$ atomic layers of $\mathrm{Cu}_{50} \mathrm{Co}_{50}$ alloy|semi-infinite Co. The results given in the table do not depend sensitively on the alloy concentration used.

For a clean $\mathrm{Cu} / \mathrm{Co}(111)$ interface, the real part of the mixing conductance is comparable in size to the spin-up and spin-down conductances but the imaginary part is almost a 
TABLE I. Interface conductances in units of $10^{15} \Omega^{-1} \mathrm{~m}^{-2}$.

\begin{tabular}{lccccc}
\hline \hline System & Interface & $G^{\text {maj }}$ & $G^{\text {min }}$ & $\operatorname{Re} G^{\text {mix }}$ & $\operatorname{Im} G^{\text {mix }}$ \\
\hline $\mathrm{Cu} / \mathrm{Co}(111)$ & Clean & 0.42 & 0.36 & 0.41 & 0.009 \\
$\mathrm{Cu} / \mathrm{Co}(111)$ & $2 \times 50-50$ alloy & 0.42 & 0.33 & 0.55 & 0.030 \\
$\mathrm{Cu} / \mathrm{Co}(1) / \mathrm{Cu}(7) / \mathrm{Co}(111)$ & Clean & 0.40 & 0.22 & 0.41 & 0.032 \\
$\mathrm{Cu} / \mathrm{Co}(1) / \mathrm{Cu}(7) / \mathrm{Co}(111)$ & $2 \times 50-50$ alloy & 0.41 & 0.21 & 0.55 & 0.036 \\
$\mathrm{Cr} / \mathrm{Fe}(001)$ & Clean & 0.14 & 0.35 & 0.61 & 0.028 \\
$\mathrm{Cr} / \mathrm{Fe}(001)$ & $2 \times 50-50$ alloy & 0.26 & 0.34 & 0.61 & 0.052 \\
\hline $\mathrm{Cu} / \mathrm{Co} / \mathrm{Vac} / \mathrm{Cu}$ & clean & $9.3 \times 10^{-12}$ & $1.9 \times 10^{-11}$ & 0.41 & -0.041 \\
$\mathrm{Cu} / \mathrm{Co} / \mathrm{Vac} / \mathrm{Cu}$ & $2 \times 50-50$ alloy & $3.3 \times 10^{-11}$ & $3.0 \times 10^{-11}$ & 0.53 & 0.003 \\
\hline \hline
\end{tabular}

factor of 50 smaller. Interface disorder increases the mixing conductance, the real part by about $35 \%$, the imaginary part by a factor of 3 .

It is interesting to compare $\left|G^{m i x}\right|$ with $G^{\uparrow}+G^{\downarrow}$ because the torque only depends on the mixing conductance while the electron current is mainly determined by $G^{\uparrow}+G^{\downarrow}$. Large values of $\left|G^{m i x}\right| /\left(G^{\uparrow}+G^{\downarrow}\right)$ mean more torque per unit current. The calculations show that disorder at the $\mathrm{Co} / \mathrm{Cu}$ interface increases the spin torque. Another possibility to increase the ratio of $\left|G^{\text {mix }}\right|$ to $G^{\uparrow}+G^{\downarrow}$ is to insert an impurity layer on the $\mathrm{Cu}$ side; a $\mathrm{Co}$ monolayer inserted on the $\mathrm{Cu}$ side scarcely changes the mixing conductance but reduces the normal conductance significantly.

For the $\mathrm{Cr} / \mathrm{Fe}$ interface the band-structure matching and the effect of interface disorder are quite different compared to $\mathrm{Cu} / \mathrm{Co}$. Whereas the majority-spin states of $\mathrm{Cu} / \mathrm{Co}$ match very well, it is the minority-spin states in $\mathrm{Cr}$ and $\mathrm{Fe}$ which match best; see Figs. 1(c,d). For a perfect $\mathrm{Cr} / \mathrm{Fe}(001)$ interface, the mixing conductance is almost twice as large as the normal conductance. The expression for the mixing conductance suggests that having a large number of propagating channels (flux-normalized right- or left-going Bloch states at the Fermi energy) on the nonferromagnetic side of the interface should lead to a large mixing conductance.

It is of interest to have a closer look at the $\mathbf{k}_{\|}$resolved mixing conductance $G^{m i x}$. We can see from Fig. 1(f) that close to the center of the BZ the real part of $G^{\text {mix }}$ is very large, even larger than the number of channels in $\mathrm{Cr}$ at the same $\mathbf{k}_{\|}$points, shown in Fig. 1(b); at the same $\mathbf{k}_{\|}$points the transmission of majority-spin electrons is very low. Thus at some $\mathbf{k}_{\|}$the mixing conductance can be much larger than the normal conductance. This can be understood in terms of a simple one-dimensional infinite barrier model in which the spin-up and spin-down barriers are displaced in space by an amount $\Delta$. For both spins the reflection amplitude is 1 . However, the displacement $\Delta$ introduces a phase shift $e^{-2 i k \Delta}$ for electrons with wave number $k$ so that the mixing conductance can have any value $G_{0}\left(1-e^{-2 i k \Delta}\right)$ for this simple single-channel model. Although $G^{\text {mix }}$ is large around $\mathbf{k}_{\|}=0$ for $\mathrm{Fe} / \mathrm{Cr}$, the minority-spin reflection is very low in most parts of the BZ so that after averaging over the BZ, $G^{\text {mix }}$ is not very high compared with the normal conductance.

The imaginary part of $G^{m i x}$ is related to the spin precession which results from the noncollinear alignment of the spins of the injected electrons and the magnetization (or an external magnetic field). A nonvanishing imaginary part of the mixing conductance, $\operatorname{Im} G^{m i x}$, should result in antisymmetry with respect to time reversal. ${ }^{20,21}$ However, $\operatorname{Im} G^{\text {mix }}$ is small in all the systems we have studied. The reason for this can be understood by examining the $\mathbf{k}_{\|}$resolved imaginary part of $G^{m i x}$ shown in Fig. 1(e). $\operatorname{Im} G^{m i x}$ can be negative as well as positive and it is the partial cancellation of these contributions which leads to the net result being small. This can be illustrated using the simple phase-shift model as follows. Suppose that the phase shift of the reflected waves $\delta\left(\mathbf{k}_{\|}\right)$is distributed randomly between $\varphi_{1}$ and $\varphi_{2}$ with equal weights and that the amplitude of $r_{\uparrow}^{\dagger} r_{\downarrow}$ is $A$. Then the average mixing conductance is $\left[G_{0} /\left(\varphi_{2}-\varphi_{1}\right)\right] \int_{\varphi_{1}}^{\varphi_{2}}\left(1-A e^{i \delta}\right) d \delta$ $=\left(e^{2} / h\right)\left\{1+\left[i A /\left(\varphi_{2}-\varphi_{1}\right)\right]\left(e^{i \varphi_{2}}-e^{i \varphi_{1}}\right)\right\}$ where $G_{0}=e^{2} / h$. If $\varphi_{1}=-\varphi_{2}$ then $\operatorname{Im} G^{\text {mix }}$ is zero and $\operatorname{Re} G^{m i x}=G_{0}$. In a more realistic treatment the weights will not be homogeneous and $\varphi_{1}$ will be slightly different from $-\varphi_{2}$ so that $\operatorname{Im} G^{m i x}$ is not exactly zero. To avoid this type of cancellation and obtain a large $G^{m i x}$, one should use as the nonmagnetic element a material in which the conducting channels occupy only a small region of $\mathbf{k}_{\|}$space, for example, a doped semiconductor. ImG $G^{\text {mix }}$ could also be significant in a ferromagnetic insulator. ${ }^{21}$

We may conclude that a large reflection amplitude does not mean that the mixing conductance must be small. It provides us with a means to realize large values of $\left|G^{\text {mix }}\right| /\left(G^{\uparrow}\right.$ $+G^{\downarrow}$ ) by using a tunnel junction or ferromagnetic insulator as the FM element. For such nonconducting interfaces we predict that it will be possible to obtain a non-zero-spin current while the electronic current is zero or vanishingly small. We confirm this by calculating the mixing conductance for a $\mathrm{Cu} / \mathrm{Co} / \mathrm{Vac} / \mathrm{Cu}$ tunnel junction. ${ }^{22}$ While $G^{\text {mix }}$ is comparable to that found for metallic systems (see Table I), the normal conductance is vanishingly small. For thicker vacuum (insulating) layers the torque simply equals that of the metallic interface and the charge current is suppressed even more.

In summary, we have studied the mixing conductance of $\mathrm{Cu} / \mathrm{Co}, \mathrm{Cr} / \mathrm{Fe}$, and $\mathrm{Cu} / \mathrm{Co} /$ Vacuum/Co configurations taking the full transition-metal electronic structure into account and including disorder. The effect of the mixing and normal conductances can be separated for a three-terminal device where one of the terminals is a ferromagnetic insulator or a magnetic tunnel junction for which the normal conductance can be made vanishingly small without affecting the size of the mixing conductance. 
We are grateful to Yuli Nazarov and Daniel HuertasHernando for useful discussions. This work is part of the research program for the "Stichting voor Fundamenteel Onderzoek der Materie" (FOM) and was supported by the Norwegian Research Council, the NEDO International Joint Research Grant Program "Nano-magnetoelectronics," the Grant Agency of the Czech Republic (Grant No. 202/00/ 0122), as well as by the European Commission's TMR Research Network on "Interface Magnetism" (Contract No. FMRX-CT96-0089) and the RT Network "Computational Magnetoelectronics" (Contract No. HPRN-CT-200000143).
${ }^{1}$ For recent reviews, see M.A.M. Gijs and G.E.W. Bauer, Adv. Phys. 46, 285 (1997); J.-P. Ansermet, J. Phys.: Condens. Matter 10, 6027 (1998); A. Barthélémy, A. Fert, and F. Petroff, in Handbook of Magnetic Materials, edited by K. H. J. Buschow (Elsevier, Amsterdam, 1999), Vol. 12; E.Y. Tsymbal and D.G. Pettifor, Solid State Phys. 56, 113 (2001).

${ }^{2}$ J.C. Slonczewski, J. Magn. Magn. Mater. 159, L1 (1996).

${ }^{3}$ L. Berger, Phys. Rev. B 54, 9353 (1996).

${ }^{4}$ K. Inomata, IEICE Trans. Electron. E84-C, 740 (2001).

${ }^{5}$ M. Tsoi et al., Phys. Rev. Lett. 80, 4281 (1998); J.-E. Wegrowe et al., Europhys. Lett. 45, 626 (1999); J.Z. Sun, J. Magn. Magn. Mater. 202, 157 (1999); E.B. Myers et al., Science 285, 867 (1999); J.A. Katine et al., Phys. Rev. Lett. 84, 3149 (2000); J. Grollier et al., Appl. Phys. Lett. 78, 3663 (2001).

${ }^{6}$ K.M. Schep, P.J. Kelly, and G.E.W. Bauer, Phys. Rev. Lett. 74, 586 (1995).

${ }^{7}$ P. Zahn, I. Mertig, M. Richter, and H. Eschrig, Phys. Rev. Lett. 75, 2996 (1995).

${ }^{8}$ X. Waintal et al., Phys. Rev. B 62, 12317 (2000).

${ }^{9}$ A. Brataas, Yu.V. Nazarov, and G.E.W. Bauer, Phys. Rev. Lett. 84, 2481 (2000); Eur. Phys. J. B 22, 99 (2001).

${ }^{10}$ K.M. Schep et al., Phys. Rev. B 56, 10805 (1997)

${ }^{11}$ M.D. Stiles and D.R. Penn, Phys. Rev. B 61, 3200 (2000).

${ }^{12}$ K. Xia et al., Phys. Rev. B 63, 064407 (2001).

${ }^{13}$ S. Datta, Electronic Transport in Mesoscopic Systems (Cambridge University Press, Cambridge, England, 1995).

${ }^{14}$ J.S. Moodera et al., Phys. Rev. Lett. 74, 3273 (1995).

${ }^{15}$ G.E.W. Bauer et al., Mater. Sci. Eng., B 84, 31 (2001).

${ }^{16}$ The elements of the transmission matrix $t$ which enter the conductances are the scattering theory transmission amplitudes used in the Landauer-Büttiker formalism to describe electrical trans- port (Ref. 13). In Refs. 10 and 12 where we reported the development of methods for calculating these matrices from first principles, they were used in an application where only the matrix of transmission probabilities $T$, obtained by squaring the complex amplitudes, $T=t t^{\dagger}$, were needed. In the present work we need $t$, as discussed in Refs. 8 and 9.

${ }^{17}$ I. Turek et al., Electronic Structure of Disordered Alloys, Surfaces and Interfaces (Kluwer, Boston, 1997).

${ }^{18}$ O. K. Andersen, O. Jepsen, and D. Glötzel, in Highlights in Condensed Matter Theory, edited by F. Bassani, F. Fumi, and M. P. Tosi (North-Holland, Amsterdam, 1985), p. 59. In our calculations we use the atomic spheres approximation (ASA) in which the atomic Wigner-Seitz cells are replaced by atomic spheres. These fill all space so there is no interstitial region. The ASA is superior to the muffin-tin approximation for close-packed metals.

${ }^{19}$ To determine charge and spin densities one needs a configurationaveraged single Green's function, a standard procedure using the CPA. Calculation of the conductance requires obtaining a configuration-averaged product of Green's functions which is much more difficult. By using supercells we obtain virtually exact results without having to compute "vertex" corrections. It has the additional advantage that we can model short-range order, steps, stacking faults, etc.

${ }^{20}$ D. Huertas-Hernando, Yu.V. Nazarov, A. Brataas, and G.E.W. Bauer, Phys. Rev. B 62, 5700 (2000).

${ }^{21}$ D. Huertas-Hernando, Yu.V. Nazarov, and W. Belzig, Phys. Rev. Lett. 88, 047003 (2002).

${ }^{22}$ In the barrier calculations reported here, six layers of empty atomic spheres were used to model the barrier. 